\title{
Index of Names (The Other Voices)
}

Adams, H. 9.QQ

Aesop 7.M

Alcuin 11.DDD

Allott, Ph. 10.F, 12.KK, 12.VV, 12.BBB, 12.EEE, 12.WWW

Allott, R.M. 8.D

Aristotle 5.W, 5BB, 6.E, 10.II, 11.YY, 12.U, 12.FFF

Arnold, M. 1.R

Augustine of Hippo 8.H, 9.K, 11.ZZ, 12.000

Avicenna (Ibn Sīnā) 3.L

Bacon, F. 1.H, 6.K, 6.L, 7.C, 7.D, 9.Y, 11.Q

Bai Juyi (Bo Juyi) 10.M

Barthes, R. 7.FF

Baudelaire, C. 9.H

Beauvoir, S. de 12.GG

Bentham, J. 9.AA, 10.W, 10.X, 10.Y, 11.D, 12.JJ

Boswell, J. 9.N, 9.Q, 9.S

Breton, A. 6.BB

Bruyère, J. de la 11.FFF

Burckhardt, J. 5.II

Burke, E. 10.E, 10.R, 11.M, 11.V, 12.GGG

Burton, R 9RR, 12.SS

Bury, J.B. 5.EE

Butler, S. 2.Q, 12.PP

Butterfield, H. 5.X

Calderón de la Barca, P. 6.A

Campbell, J. 4.S, 5.N

Camus, A 9.AAA

Carlyle, Th. 7.BB, 11.RR

Carpenter, W.B. 2.M

Carroll, L. 6.Z, 7.I, 9.W, 9DD

Cassirer, E. 1.K, 5.II, 9.F

Cervantes, M. de 6.C, 6.D

Chaucer, G. 9.LL
Chuang-tzŭ (Chuang Chou) 3.B, 4.K

Churchill, W.S. 12.AAA

Cicero, M.T. 9.A, 10.G, 12.000

Clausewitz, C. von $4 . Z$

Clerk Maxwell, J. 2.L

Clifford, W.K. 11.H

Coke, E 10.S

Coleridge, S.T. 5.E, 6.CC, 6.FF, 12.UU

Commonwealth Act 1649 11.X

Comte, A. 7.CC

Condorcet, M. de 5.J

Confucius 9.MM

Cornford, F 7.R

Cosmides, L. 4.F, 8.B

Cowper, W. 4AA

Craik, K. 7.W

Cudworth, R. 4.C

Curtius, Q. 11.AAA

D'Alembert, J. le R. 7.D

Dante Alighieri 6.T, 12.BBBB

Darwin, Ch. 11.WW

Deleuze, G. 11.QQ

Democritus of Abdera 6.S, 7.P, 12.A

Descartes, R. 7.B, 7.C

Dewey, J. 7.L, 9.EE, 11.K

Diderot, D. 9.P, 11.EEE

Dostoevsky, F. 2.G, 6.C, 9.L

Durkheim, E. 4.O, 11.F

Eccles, J. 4.B

Eckermann, J.P. 8.P

Einstein, A. 2.X, 7.T

Eliot, T.S. 5.F, 12.NN

Ellesmere, Lord 10.T, 10.U

Engels, F. 4.V, 5.O, 5.P, 5.S, 11.O, 11.VV, 12.F

Epicurus 12.D

Erasmus, D. 4.Y

Eribon, D. 7.EE 
European Human Rights Convention 10.L

European Union Treaty 12.MMM

Fanon, F. 12.W

Fichte, J.G. 8.N, 12.ZZ

Fontenelle, B. de 1.L

Foucault, M. 7.EE

Frankfort, H. 2.O, 4.R

Frazer, J.G. $10 . \mathrm{O}$

Free Pardon Act 1660 11.Y

Freud, S. 2.Y, 6.F, 7F, 7.K, 7.Z, 8.M, 8.Q, 11.I, 12.K

Fung Yu-Lan 12.ZZZ

Fustel de Coulanges, N. 1.I, 11.G

Galen 7.P

Gibbon, E. 5.G

Gide, A. 8.J, 9.UU

Giorgione 6.GG

Gobineau, A. de 12.V

Godwin, W. 11.A, 12.LL

Goethe, J.W. von 8.P

Grant, M. 5.GG

Green, J. 8.J

Guattari, F. 11.QQ

Haeckel, E. 4.P

Hall, W.E. 10.FF

Harrison, J.E. 6.DD, 9.WW

Hegel, G.W.F. 1.J, 3.E, 4.G, 5.I, 5.Y, 7.AA, 9.YY, 10.EE, 12.B, 12.Q, 12.TTT

Heraclitus 2.A, 3.A, 9.F

Herbert, G. 9.BBB

Herodotus 5.FF

Hintze, O. 5.V

Hitler, A. $12 . \mathrm{KKK}$

Hobbes, Th. 4.W, 5.K, 8.E, 10.C, 11. $\mathrm{R}$

Holmes, O.W. 10.V

Horace 8.V

Hsun Ch'ing 9.J

Hugo, V. 8.L

Hui-ssu 8.O, 9.G

Huizinga, J. 6.V

Humboldt, W. von 12.M

Hume, D. 4.M, 7.Y, 8.F, 10.N, 12.XX

Huxley, J. 12.UUU

Huxley, T.H. 7.G
Ibn Sīnā 3.L

Ibsen, H. 12.CC

James, W. 3.E, 8.C, 11.H

Jastrow, J. 4.J

Jefferson, Th. 10.J, 11.FF

Johnson, S. 9.N, 9.Q, 9.S

Jung, C.J. 2.K, 7.GG, 9.M

Kant, I. 8.W, 9.T, 11.U, $12 . \mathrm{O}$

Katha Upanishad 3.G

Keats, J. 1.O, 7.J

Kellogg-Briand Pact 12.JJJ

Kelsen, H. 10.Z

Keynes, J.M. 11.NN

Kierkegaard, S. 9.OO

Kipling, R. 12.DDDD

Lamartine, A. de 2.U

Lassalle, F. 11.P

Lasswell, H.D. 10.HH

Lawrence, D.H. 12.H

League of Nations Covenant 12.III

Leibniz, G.W. 1.G, 1.L, 4.D, 8.T

Liberties of Massachusetts 10.JJ

Lipsius, J. 9.RR

Locke, J. 4.X, 7.X, 7.HH, 8.T, 9.B, 9.R, 10.I

Lucretius 7.0

Lundstedt, V. 10.AA

Macaulay, Th. 7.E, 11.N

McDougal, M.S. 10.HH

Mach, E. 7.Q

Machiavelli, N. 1.C, 11.N

McLuhan, M. 2.U

Madison, J. 10.J, 12.Y

Malthus, Th. 11.OO, 11.UU, 11.WW

Mandeville, B. de 11.II

Marcus Aurelius 3.I, 12.LL, $12 . \mathrm{OOO}$

Marcuse, H. 9.JJ, 11.XX

Martial 9.D

Marvell, A. 8.K

Marx, K. 4.V, 5.O, 5.P, 5.S, 9.KK, 11.O, 11.KK, 12.F, 12.FF

Massachusetts Constitution 11.T

Michelet, J. 11.E

Mill, J.S. 5.Q, 12.N, 12.X 
Mises, L. von 11.LL

Mo Ti (Mo Tzŭ) 3.N, 10.H

Montaigne, M. de 7.A, 7.C, 9CC

Montesquieu, Ch. de 5.M, 10.A, 11. $\mathrm{HH}$

More, Th. 1.E, 5.T, 9.TT, 10.U, 12.JJ, 12.WW

Morgenthau, H. 10.GG

Morris, C. 12.L

Mozart, W.A. 6.HH

Mundaka Upanishad 3.J

Murasaki, S. 6.EE

Murray, G. 11.J

Mussolini, B 11.CCC

Newbolt, H. 6.W

Newman, J.H. 12.NN

Newton, I. 7.N

Nietzsche, F. 1.N, 2.W, 4.H, 4.I, 7.DD, 8.R, 9.XX, 9.ZZ, 11.H, 12.E, 12.J, 12.DD, 12.CCCC

Novalis (F. von Hardenberg) 1.Q

Nishida, K. 12.XXX

Oblivion Act 1660 11.Y

Ogden, C.K. 4.N

Olivecrona, K. 10.BB

Ortega y Gasset, J. 6.I, 12.R

Ovid 3.M

Owen, R. 11.B, 12.HH, 12.VVV

Paine, Th. 11.L, 11.CC

Pascal, B. 2.D, 2.I, 2.N, 3.D, 8.G, 11.YY, 12.G

Pericles 5.AA, 12.S

Peri Rossi, C. 1.P

Philodemus 6.S

Pico della Mirandola, G. 2.F

Pilgrim Fathers 11.W

Plato 2.T, 3.C, 3.K, 3.T, 4.L, 4.U, 6.G, 6.H, 6.U, 8.A, 9.C, 9.U, 9.X, 9.GG, 9.SS, 10.D, 11.YY, 11.BBB, 12.T, 12.DD

Pope, A. 2.E, 12.AAA

Popper, K. 5.R

Pott, H. 6.K

Pound, R. 10.CC

Proudhon, P-J. 11.DD

Quine, W. 9.FF
Ranke, L. von 5.DD

Reid, Th. 11.C

Renan, E. 5.D, 12.YY

Richards, I.A. 4.N

Richter, J.P.F. ('Jean Paul') 2.J

Riesman, D. 12.P

Rimbaud, A. 12.R

Rorty, R. 9.GG

Rousseau, J.-J. 1.B, 2.H, 3.F, 5.JJ, 8.I, 10.B, 11.S

Ruskin, J. 5.U, 6.N, 6.O, 11.SS, 12.TT

Russell, B. 9.BB

Ryle, G. 4.A

Saint-Simon, H. de 9.VV

Schiller, F. 2.R, 5.E, 11.PP

Schopenhauer, A. 1.G, 2.B, 5.B, 6.B, 6.M, 6.P, 6.R, 9.O, 11.H, 12.I, 12.J, 12.NNN

Shaftesbury, Earl of 8.U

Shakespeare, W. 1.A, 1.M, 6.B, 6.C, 6.J, 6.AA, 8.S, 9.V

Smith, A. 7.V, 11.X, 11.GG, 11.GGG

Smith, Th. 10.P

Socrates 2.T, 3.C, 3.N, 4.L, 6.U, 9.C, 9.SS, 10.D

Sophocles 2.C, 4.T

Spencer, H. 6.X

Spinoza, B. 2.P, 4.E

Steiner, G. 6.T

Suárez, F. 12.QQQ

Sumner, W.G. 1.F

Swift, J. 10.Q, 12.QQ

Taine, H. 5.CC

Takahama, K. 12.EEEE

Tâo Tê Ching 3.H, 12.II, 12.YYY

Teilhard de Chardin, P. 12.UUU

Tertullian 7.H

Thucydides 5.AA, 12.S

Tocqueville, A. de 2.S, 2.V

Tolstoy, L. 5.C, 5.KK, 11.TT, 12.C, 12.BB, 12.CCC, 12.DDD

Tooby, J. 4.F, 8.B

Treaty of Paris 1783 11.AA

Trevelyan, G.M. 5.HH

Turgot, A.R.J 11.MM 
U.N. Charter 12.LLL

Universal Human Rights Declaration 1948 10.K

U.S. Constitution 1787 12.HHH

U.S. Declaration of Independence 1776 $11 . \mathrm{Z}$

Valéry, P 5.A, 9.HH, 9.PP, 12.SSS

Vattel, E. de 10.DD

Veblen, T. 6.Q

Vico, G. 5.Z, 9.E

Vitoria, F. de 12.PPP

Voltaire 1.D, 5.H, 9.I
Waugh, E. 5.L

Weber, M. 11.BB, 11.EE, 11.JJ, 12.Z

Whewell, W. 12.RR

Whitehead, A.N. 7.S, 9.II

Wilde, O. 9.NN

Wilson, E.O. 4.Q

Wittgenstein, L. 9.Z, 9.BB

Wodehouse, P.G. 6.Y

Wolff, C. von 12.RRR

Wollstonecraft, M. 12.EE

Wright Mills, C. 12.AA

Zaehner, R.C. 9.M 